\title{
LE TERRITOIRE ENTRE ESPACE ET POUVOIR : POUR UNE PLANIFICATION TERRITORIALE ASCENDANTE
}

\author{
Patrick d'Aquino
}

Belin | « L’Espace géographique »

2002/1 tome 31 | pages 3 à 22

ISSN 0046-2497

ISBN 270113126X

Article disponible en ligne à l'adresse :

https://www.cairn.info/revue-espace-geographique-2002-1-page-3.htm

Distribution électronique Cairn.info pour Belin.

(C) Belin. Tous droits réservés pour tous pays.

La reproduction ou représentation de cet article, notamment par photocopie, n'est autorisée que dans les limites des conditions générales d'utilisation du site ou, le cas échéant, des conditions générales de la licence souscrite par votre établissement. Toute autre reproduction ou représentation, en tout ou partie, sous quelque forme et de quelque manière que ce soit, est interdite sauf accord préalable et écrit de l'éditeur, en dehors des cas prévus par la législation en vigueur en France. Il est précisé que son stockage dans une base de données est également interdit. 


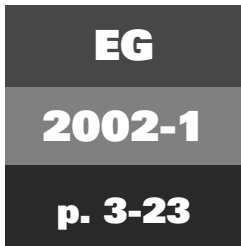

\section{Le développement local Le territoire entre espace et pouvoir : pour une planification territoriale ascendante}

\section{PATRICK D'AQUINO}

Géographe, CIRAD, BP 175, Saint-Louis, Sénégal, daquino@cirad.fr
RÉSUMÉ .- Le poids des acteurs locaux sur les politiques de développement reste limité, y compris au sein des démarches dites participatives. L'amélioration de la distribution des pouvoirs entre encadrement technique et société civile est un enjeu fondamental, pour éviter les blocages, les replis identitaires et les utopies du « village planétaire ». Le géographe devrait intégrer la dimension politique des territoires dans sa réflexion, et jouer un rôle clé dans l'émergence d'un nouveau type de processus démocratique, du local vers le global. Nous proposons, sous le nom de planification territoriale ascendante, une dynamique réellement endogène et interscalaire. Installer une dynamique locale implique alors trois hypothèses : le principe d'endogénéité des dynamiques locales, le principe de permanence de la démarche de planification, le caractère normalement ascendant d'une planification continue.

DÉCENTRALISATION, DÉMOCRATIE, DÉVELOPPEMENT LOCAL, PARTICIPATION, PLANIFICATION, TERRITOIRE
ABSTRACT.- Territorial development, between space and power: an argument for bottom-up territorial planning.The influence of local actors on development policies remains limited, including in so-called participatory approaches. The distribution of power between technical expertise and civil society must be improved to avoid blockages, with drawal into identity groups and utopian ideas of the "global village». The geographer must integrate the political dimension of territories into his thinking and play a key role in the emergence of a new type of democratic process, from local to global. With bottom-up territorial planning, we propose a genuinely endogenous, inter-level dynamic. Setting up a local dynamic is based on three assumptions: local dynamics must be endogenous; the planning approach must be continuous; and this continuous planning must be mostly bottom-up.

DECENTRALISATION, DEMOCRACY, LOCAL DEVELOPMENT, PARTICIPATION, PLANNING, TERRITORY
A u Nord comme au Sud, il est maintenant admis que la mondialisation ne pourra se passer d'une certaine reconnaissance du " local " et des responsabilités qu'il prendra dans son propre développement. Cependant, même si les expériences des vingt dernières années ${ }^{1}$ ont donné corps à des approches locales des problématiques de développement, ces expérimentations localisées peinent encore à se déployer en une dynamique régionale et durable. On sait concevoir des outils d'analyse pour une gestion décentralisée, on sait élaborer des cadres réglementaires et législatifs, mais on ne réussit guère que des expériences localisées dans le temps et l'espace. La difficulté est de parvenir à généraliser des dynamiques durables, à les ancrer dans les pratiques des habitants et des institutions.

1. Cf. les approches participatives, le développement local, les politiques de décentralisation (Pecqueur, 1989; Chambers, 1993; Vachon, 1993; Houée, 1996; D’Aquino à paraître). 
2. Notre travail se fonde en partie sur les résultats d'une opération pilote d'appui expérimental à la décentralisation, menée entre 1996 et 2000 dans la vallée du fleuve Sénégal. L'expérimentation a associé le Centre international de recherches agronomiques pour le développement (CIRAD, Montpellier, France), la Société nationale d'aménagement des terres du delta du fleuve Sénégal et des vallées du fleuve Sénégal et de la Falémé (SAED), I'Université Gaston Berger (UGB, Saint-Louis, Sénégal), I'Institut Sénégalais de Recherche Agronomique (ISRA, SaintLouis, Sénégal) et les collectivités locales rurales et régionales de la vallée du fleuve Sénégal. Elle avait pour objet de concevoir, tester et finaliser différents supports méthodologiques opérationnels (tels que la carte, le SIG, le jeu de rôles et les systèmes de simulation multiagents) pour une réussite durable des dynamiques de décentralisation et de développement local à différentes échelles (terroirs, espaces locaux de $3500 \mathrm{~km}^{2}$, région de $18000 \mathrm{~km}^{2}$ ). Certains passages de cet article ont pu figurer dans le rapport de soutenance pour mon habilitation à diriger des recherches.
En outre, le développement localisé d'un territoire impose de nouvelles formes de relations entre les services d'appui et les populations, qui doivent maintenant maitriser toutes les étapes du processus de décision sur leur espace. Cette mutation, inéluctable, reste peu engagée. Les analyses d'experts impliquent au préalable des choix de développement, en amont de ce qui devrait être une concertation interne et autonome sur les options futures de développement du territoire. Quelle que soit la méthode de concertation employée, ses principes sont dévoyés, même inconsciemment, par des intervenants qui sont instinctivement portés à prendre les décisions à la place des acteurs concernés.

D'un côté, autant en matière de développement durable que de démocratie locale, l'amélioration de cette distribution des pouvoirs entre encadrement technique et société civile paraît un enjeu fondamental de ces prochaines années. De l'autre, « local » est partout conjugué avec " territoire » selon des déclinaisons autant passéistes (les replis identitaires) qu'utopistes (le village planétaire). Il est temps que la géographie réinvestisse la politique locale, ou plutôt localisée, pour une conjugaison mieux adaptée au temps présent et à ses dynamiques² ${ }^{2}$

\section{Démocratie locale et développement}

\section{Expliciter le politique}

Tout programme de développement apporte son idéologie politique implicite. En particulier, toute intervention en faveur d'une gestion locale de territoire contient une certaine volonté de changement politique. Exclure le politique de l'analyse amènerait à « tomber dans un angélisme complètement irréaliste " (Friedberg, 1993). Quelle que soit l'approche, elle aspire à l'installation d'un processus local de décision le plus participatif, le plus large ou le plus juste possible. Même les économistes reconnaissent que le développement régional est avant tout une question éthique et politique (Weaver, 1983, p. 180), une "intention politique de la recherche du bien commun " (Mercier, 1989, p. 70).

On doit savoir reconnaître et expliciter les choix politiques sous-entendus par l'intervention, et ce qu'implique le concept même de développement territorial décentralisé, quitte à réhabiliter la dimension politique dans la gestion locale des ressources (Calame, 1994, p. 8), au lieu de s'en tenir à un habituel silence. La science du développement, ni libre entreprise ni action neutre, doit être précise sur ses desseins, être claire sur ses biais et ses hypothèses préalables (Rosenau, 1990). Cela fait partie du travail scientifique que de poser des questions sur la nature même du regard dit scientifique (Bourdieu, 1994, p. 225), d'alerter les interlocuteurs et de juger avec eux de la pertinence de ces choix.

\section{Le développement local, sans garantie démocratique}

Dans le cas de l'appui à une gestion locale, ces choix politiques constituent même les finalités profondes des approches mises en œuvre, sans guère explicitation ni débat avec les acteurs concernés et les responsables politiques locaux. Ainsi, les tenants du développement local et des approches participatives ont le plus souvent pour idéologie, consciente ou inconsciente, l'approche étatsunienne du pouvoir local, et la société civile est leur championne. Cette approche est née aux États-Unis à partir de travaux sociologiques des années 1950 qui ont souligné le rôle des réseaux informels notabiliaires dans les prises de décision politique (Hunter, 1952; Mills, 1956; Frakenberg, 1966; 
Dahl, 1971; Remy, 1988). Ce « jeu social invisible " (Dahl, 1971), en coulisse ${ }^{3}$ de la scène publique locale, faussait le jeu démocratique: l'approche " communautariste " (community organization movement) naquit de la volonté de créer un contre-pouvoir local et populaire, qui aurait une base territoriale (Coleman, 1961). Lié à l'idée d'un changement social à assumer et à orienter, le modèle de la community est profondément antipolitique et anti-institutionnel: c'est "l'antithèse de l'État ", la finesse des dynamismes sociaux s'opposant à la "mollesse " de ses formes institutionnelles (Grémion, 1976, p. 264). L'acteur-citoyen, hostile à l'interventionnisme de l'État, s'investit dans la vie associative, le self-help (Katz, 1993) et les réseaux d'influence (Lassale, 1991). L'État et l'administration doivent sans cesse faire la preuve de leur légitimité et de leurs compétences face à d'autres légitimités concurrentes, et sont constamment tenus de rendre compte à leurs mandants (accountability ${ }^{4}$ ).

Il y a dans cette perception de la démocratie et du pouvoir local un choix idéologique d'autorégulation sociopolitique, comme en économie ${ }^{5}$ : c'est la "société automatique " que décrit Tocqueville. La décision politique y est considérée comme un équilibre "raisonné " (Grémion, 1976, p. 265) du pouvoir entre les groupes. La théorie du pouvoir qui lui est liée est davantage une théorie de l'influence, principe constitutionnel des checks and balances, orgueil de la démocratie américaine: "C’est là sa valeur et sa limite " (Ibid.). Le community power est un mode de mobilisation sociale exemplaire, inspirant les concepteurs des approches participatives. Mais ce modèle est critiquable en tant que mode de gouvernement car il contient, par définition, les mêmes vices que les réseaux notabiliaires qu'il veut combattre. Les groupes de pression " citoyens » ont été construits sur le même modèle que ces réseaux, sans contrepartie visible sur la scène publique, celle-ci étant plus ou moins désinvestie et dévalorisée par les différentes parties. Ici aussi tout se passe en coulisse, où les processus de négociation permettent à des intérêts structurés, "citoyens " ou notabiliaires, de jouer un rôle disproportionné (Long, 1958).

«Le modèle se rapproche [...] de la perfection si la distribution du pouvoir [...] est assez fluide pour permettre une participation ouverte des différents intérêts de la communauté » (Grémion, 1976, p. 246), ce qui n'arrive guère: la démocratie directe est une utopie dangereuse, car elle empêche de rechercher une voie plus indirecte mais plus réaliste. On laisse tomber l'opinion majoritaire au profit d'un pouvoir d'influence opposé à toute régulation institutionnelle. Le libéralisme social s'activant en coulisse ne produit pas de choix collectif de valeurs explicite et visible et ne garantit donc pas le respect de ces valeurs. La lacune de l'approche américaine du community power est qu'elle ne contient aucune garantie sur les valeurs démocratiques. La "société automatique " de Tocqueville n'est pas automatiquement démocratique. En refusant toute institution formelle de concertation et de décision collective, on écarte la possibilité d'un choix explicite et le respect de valeurs démocratiques. Pourtant, c'est ce modèle américain du community power, fondamental pour comprendre la perception américaine de la démocratie (Grémion, 1976, p. 264), en particulier locale, qui a profondément influencé la conception du développement local.

\section{Les approches participatives, issues du communautarisme libéral}

Il y a souvent une ambiguité non tranchée, voire une opposition, entre les tenants du développement local et participatif et les politiques de décentralisation. Beaucoup des adeptes du développement local restent détachés et méfiants vis-à-vis des collectivités territoriales décentralisées, pouvoir politique local institué par un État central. Pour eux,
3. Cf. le paradigme de Goffman distinguant la scène et les coulisses dans I'environnement politique américain (Remy, 1988).

4. Idée selon laquelle ceux qui exercent le pouvoir à quelque niveau que ce soit (gouvernants, élus, nommés) ont l'obligation de rendre compte de leur action à leurs mandants (les citoyens) et de leur fournir la preuve de la conformité de leur action aux normes morales ou juridiques qui doivent la régir (Hermet et al., 1994, p. 8-9).

5. La "main invisible" d'Adam Smith. 
la valorisation des initiatives locales, la reconnaissance et la participation des populations impliquent la remise en cause du rôle des institutions, même locales, dans la régulation et la gestion des affaires locales. L'institution représente un obstacle à un lien social plus harmonieux et libéré, elle est vue comme un instrument forgé par certains groupes sociaux pour assurer leur domination sur les autres. Ainsi, dans l'appui occidental aux pays en voie de développement, les intervenants les plus progressistes écartent souvent les collectivités locales, soupçonnées de dérives politiques, au profit des organisations coutumières, qui ne sont pas toujours ni plus participatives, ni plus équitables ou démocratiques (Ela, 1982; Magassouba, 1984), mais qui ont le bénéfice exotique de l'" authenticité ». Lorsque le développement local coexiste avec la décentralisation, la gêne s'installe donc, les positionnements restent flous (Territoires, 1996; CFD, 1998).

La question fondamentale est celle de l'intérêt général. Dans l'approche du community power, il est défini à partir d'un compromis où l'État n'est qu'un des acteurs parmi d'autres (Lassale, 1991). De fait, la participation citoyenne "à l'américaine " (Lassale, 1991; Katz, 1993) signifie surtout défendre efficacement son action (y compris de contrôle), donc son intérêt particulier. La perception traditionnelle française, d'où sont issues les politiques de décentralisation, s'attache plutôt à l'adhésion à des valeurs collectives (équité, justice), qui sont garanties par les institutions: en France, l'intérêt général est ainsi défini par un arbitrage du pouvoir central, à qui est délégué le droit d'imposer " une égalité de traitement abstraite " (Lassale, 1991). La citoyenneté se résume alors à l'élection de représentants et à une pression conjoncturelle, souvent désordonnée (grèves). C'est une forme de démocratie strictement représentative, voire simplement procédurale (Sandel, 1984), "où les élus s'expriment au nom du peuple qui n'a pour faculté que de les désigner et de les révoquer périodiquement " (Hermet, 1994, p. 80). La foi en la toute-puissante justice des institutions y montre alors ses limites. Contrairement au community power, il y existe une certaine méfiance envers les initiatives "désordonnées " d'une société civile non contrôlée et l'énergie des acteurs locaux est alors contrainte et canalisée « dans des constructions préétablies et rationalisées limitant leur autonomie" (Lassale, 1991).

Il peut donc y avoir des interprétations sensiblement différentes des notions de citoyenneté, de valeur démocratique et de démocratie locale. Même en restant très pragmatique, une opération en faveur d'une gestion locale du territoire ne peut ignorer ce débat: ces choix politiques implicites ou explicites sont l'essence même de l'intervention et motivent les comportements de tous les acteurs concernés, en particulier des intervenants. D'un côté, la remise en cause des institutions régulatrices garantes des valeurs démocratiques, d'un autre, la dévalorisation du droit à la décision des citoyens: ces deux idéologies opposées sont toutes deux dangereuses pour l'avenir de la démocratie locale et sont à l'origine des ambiguiités qui polluent les interventions en faveur d'une gestion locale du territoire et d'un développement local. Si la toute-puissance jacobine des institutions a montré depuis longtemps ses déficits démocratiques, le recours inverse à la seule "société civile " n'est pas fondamentalement une garantie plus démocratique. De plus, cette opposition manichéenne contribue à développer une fracture grave entre société civile et élus; il peut être très dangereux de faire l'impasse sur une représentation démocratique de la population et l'établissement de forums institutionnalisés garantissant des valeurs collectives, au nom d'une méfiance et d'un libéralisme politiques qui risquent fort de se 
transformer en anarchie ou en communautarisme xénophobe. Une nouvelle approche plus modérée semble alors utile, qui cherchera clairement à rapprocher société civile et élus au lieu de les opposer, en renforçant une entité de régulation: le territoire.

\section{Pour une nouvelle approche des territoires}

La démocratie n'a de sens que si elle implique la gestion ou l'association de la grande majorité des personnes soumises à l'autorité représentative (Human et Zaaiman, 1996). Les conceptions américaines du pouvoir citoyen (accountability) ne seront bénéfiques que si elles sortent des coulisses, avec un arbitrage au grand jour garantissant le respect des valeurs collectives et de ce que l'on appellera ici la "pondération démocratique ", c'est-à-dire la garantie que l'opinion de la majorité de la population, et non pas de groupes minoritaires influents, a été prise en compte. Pour Jürgen Habermas comme pour Norberto Bobbio, l'authenticité de la démocratie se mesure moins à la nature formelle de ses institutions qu'à la qualité d'un espace de débat précédant les décisions, impliquant l'ensemble de la société civile (Badie, 1997, p. 19).

\section{Le territoire, espace de débat entre réseaux sociaux}

La crise de la société moderne nous interroge sur la nécessité de lier plus étroitement monde politique et société civile. Investir la scène publique et renforcer le pouvoir de celle-ci vis-à-vis des réseaux ${ }^{6}$ implique de confier l'organisation du débat aux collectivités locales, plutôt que de les marginaliser. Tous les citoyens doivent donc pouvoir s'exprimer au sein d'un "espace de citoyenneté $»^{7}$ légitimé explicitement, qui implique le citoyen dans tout le processus de prise de décision des élus. En ce sens, seul un territoire réhabilité peut jouer efficacement ce rôle d'espace de citoyenneté. Ce territoire est alors un construit social (Pellegrino et al., 1983; Di Méo, 1998), à forte teneur institutionnelle et politique ${ }^{8}$. Espace d'une dynamique sociale, il ne peut certes se restreindre à « la vision cartésienne d'un espace hiérarchique, mesurable, borné et délimité par de multiples frontières" (Bonnemaison et al., 1997) ; mais la référence spatiale y est centrale. C'est ce lien spatial qui procure au territoire un statut irremplaçable d'espace de citoyenneté et de garant de la "pondération démocratique ».

La référence du territoire n'est alors pas dans sa limite, mais dans la proximité spatiale. Les réseaux et les organisations territoriales se distinguent des autres par la proximité physique entre leurs éléments. Seule cette relation de proximité spatiale est synonyme d'une diversité sociale, d'une pluralité d'enjeux et d'intérêts qui replacent la prise de décision dans une arène démocratique, contrairement aux réseaux aspatiaux rassemblant de mêmes familles d'acteurs. Il ne s'agit pas ici de remettre en cause la valeur croissante, voire prépondérante, de ces réseaux sociaux, mais de souligner la spécificité du lien spatial, territorial, sa capacité de rassembler des groupes sociaux différents, essentielle pour conserver un fonctionnement démocratique au processus de décision sur un territoire. Certes, les systèmes de relations et les réseaux aterritoriaux permettent souvent que s'expriment les énergies locales (Allaire, 1988), mais c'est le territoire, site primordial de la souveraineté démocratique (Manin, 1985; Cohen, 1989; Miller, 1992; Lévy, 1996), qui permettra le débat entre ces réseaux rassemblés par une proximité spatiale. En accord avec Max Weber ${ }^{9}$, ce type de proximité est considéré ici comme la forme la plus démocratique, rassemblant une diversité des points de vue réunis dans une finalité commune, la
6. C'est sûrement l'un des grands enjeux d'une géographie de l'action territoriale.

7. Pour reprendre une expression introduite récemment dans les débats sur la décentralisation française : Territoires, janvier 1996; Loi d'orientation sur I'aménagement du territoire (LOADT, février 1995).

8. II existe de multiples approches du territoire et autant de définitions, le concept ayant été intégré par de nombreuses disciplines. Ainsi, notre propre approche du territoire est à replacer dans le cadre d'une problématique spécifique, consistant à définir le cadre conceptuel d'une action en faveur d'un processus démocratique pour la gestion de l'espace.

9. Le territoire est un mode d'accomplissement de la fonction de domination plus dépersonnalisée que les autres (liens personnels, communautaires, tribaux), donc un élément décisif de modernité politique d'après Max Weber (Hermet et al. 1994), qui I'oppose au modèle " patrimonial » (domination traditionnelle fondée sur le pouvoir absolu et personnalisé du chef). 
10. Cette proximité spatiale politisée, qui distingue notre territoire des autres types d'organisations sociales, politiques ou économiques, n'est cependant pas synonyme de proximité sociale, " structurale " (Gallais, 1976) ou identitaire. Elle n'est que la première étape de la construction d'un " nouveau " territoire, via un processus progressif d'émergence d'une identité territoriale: I'appropriation territoriale (D'Aquino, à paraître). prise en charge de la politique locale ${ }^{10}$. L'enjeu fondamental est de trouver un équilibre entre démocratie participative et démocratie représentative en s'appuyant sur ce territoire, qui rassemble les individus par leur proximité spatiale et permet le débat dans une diversité sociale.

\section{Vers une nouvelle forme de lien territorial entre le local et le global}

Pour améliorer la connexion à établir alors entre société civile et élus, le nouveau concept de démocratie continue (Rousseau, 1996) pourrait être une partie de la réponse. Il stipule que les procédures de la démocratie locale sont à inventer, ou plutôt à ré-inventer, dans chaque milieu, à partir de principes constitutifs de légitimité, de responsabilité et d'association des populations susceptibles de réduire l'opacité de la délégation de pouvoirs et d'assurer la correspondance entre les valeurs assumées par les mandants et celles de leurs mandataires (Hermet, 1994, p. 8-9; M. Dinet in Territoires, 1996, p. 34). Dans la même ligne de pensée que les politologues post-développementistes, l'objectif est de créer progressivement les conditions de l'« invention politique " (Badie, 1994), permettant en particulier à chacun des acteurs sociaux, même le plus humble, de négocier son devenir (Olivier de Sardan et Jaffré in Olivier de Sardan et Paquot, 1991).

Ce concept est distinct de la démocratie directe, qui abolit toute distinction entre représentants et représentés, et de la démocratie représentative, qui soustrait l'organe représentatif aux regards (Rousseau, 1996). La démocratie continue invite à une dynamique permanente de construction de la démocratie locale, rassemblant la société civile et les représentants des populations autour d'un projet et d'un apprentissage démocratiques. "La démocratie n'est pas un état permanent qu'on atteint une fois pour toutes " (Vachon, 1993, p. 101) et il ne suffit pas de doter un pays d'une constitution démocratique pour qu'il devienne effectivement une démocratie. Cet apprentissage citoyen repose sur le rapprochement de la société civile et des institutions politiques. C'est une démocratie "participative " (Barber, 1997), qui s'enracine dans les espaces publics, quels qu'ils soient (forums, associations de quartier). Elle appelle à construire une relation de confiance et de responsabilités partagées entre les élus, l'État et la société civile, au lieu de les dresser les uns contre les autres.

Il s'agit de reconstituer le tissu social déchiré par la rupture entre l'État et la société civile, par l'absence de valeurs de référence et d'un projet de société, ce que la décentralisation procéduriale actuelle ne réussira pas. Le choix politique que nous revendiquons est de viser l'installation d'un "fonctionnement démocratique " des prises de décision concernant la gestion d'un territoire infranational. Mais cela n'indique pas le transfert d'une forme d'organisation donnée. Si les principes démographiques nous paraissent universels, leurs formulations politico-administratives, coutumières ou modernes, sont plurielles, s'adaptant à chaque lieu (Barber, 1997). La démocratie est élargie à tout processus collectif de décision, coutumier ou moderne, qui permet à la population d'effectuer ensemble un choix explicite de valeurs et de participer ensuite activement à la gestion des affaires.

D'abord espace politique local, le nouveau territoire deviendrait ainsi un espace institutionnel de régulation à partir de ces choix politiques premiers. Bien que la vigilance permanente et le dynamisme des groupes sociaux de base soient indispensables, la médiation institutionnelle n'est pas à proscrire. Une certaine organisation sociale formelle, l'institution, est indispensable, comme médiatrice entre les rapports de force 
et pour aboutir à une harmonisation ou à un compromis local sur des valeurs collectivement reconnues. On s'appuie ici sur le concept d'institutionnalisation, employé par la science politique développementiste (Huntington, 1968; March et Olsen, 1990; Badie, 1994), c'est-à-dire désignant l'institution comme une condition des processus de développement et de modernisation politique d'une société. L'efficacité de ces instances collectives de décision repose sur la reconnaissance de leur légitimité, fruit de négociations et de compromis qui aboutissent progressivement à une convention (de Montgolfier et Natali, 1987; Boltanski et Thévenot, 1990; Weber et Reveret, 1993).

L'émergence de nouveaux territoires locaux cherche à favoriser la légitimité et la valeur démocratique d'un mode de gouvernement du territoire, en développant les concertations entre l'acteur et son représentant sur les valeurs collectives et sur la définition de l'intérêt général pour chaque thème abordé (D'Aquino 1998, 2000 et 2001). Il s'agit de réhabiliter le sentiment de responsabilité, que ce soit au niveau des populations, des intermédiaires ou des élus locaux (Pizzorno, 1990; Barber, 1997), par une forme d'animation institutionnelle qui cherche à créer un environnement institutionnel, un champ (Bourdieu, 1988) d'interactions, amenant les différents acteurs à un comportement démocratique. Le territoire y est en quelque sorte un "champ démocratique ", qui favorise l'apparition de comportements citoyens chez les populations et leurs différents représentants, en renforçant les liens et les interactions entre individus, en développant l'expression d'un nouvel habitus commun, favorable à la connexion entre local et global.

\section{Le géographe dans l'action territoriale}

La dimension politique est ainsi réhabilitée. Toute démarche d'appui à une gestion décentralisée des ressources constitue un processus de changement social, dans lequel exclure le politique de l'analyse amènerait à « tomber dans un angélisme complètement irréaliste " (Friedberg, 1993). Il faut revenir à cette "idée simple " (Badie, 1997, p. 19): la politique, meilleur moyen de transcender les cultures, est la science de la coexistence entre les hommes dans leur diversité. Un nouvel agencement est à découvrir, s'appuyant sur une nouvelle fondation du politique, apte à intégrer la totalité et l'individu et dans lequel la démocratie doit puiser la force d'inventer ses nouvelles formes.

L'enjeu de notre approche géographique du territoire est bien de participer à cette " invention " politique et institutionnelle. Il justifie de nos jours, pour le géographe, une certaine idéologie de l'action, dans une ambiance d'affaiblissement de la notion de nation et de territoire national, de confusion des esprits sur les territoires infranationaux et d'intrusion du mondial dans le local. Derrière cette crise du territoire, qui fait naître en chacun de nous des polarisations opposées, entre repli identitaire et aspiration au village planétaire, se profile un risque pour la démocratie, la liberté et l'égalité de l'homme. Cette situation plaide pour une géographie politique de l'action territoriale qui, en fournissant un cadre conceptuel géopolitique et en aidant à la conception de méthodes d'intervention, puisse faciliter la transformation des structures politico-administratives et passer d'une décentralisation procéduriale à de nouveaux territoires d'une démocratie locale.

Est-ce encore de la géographie? Si l'enjeu ne peut être considéré comme uniquement géographique, le sujet (le territoire), l'objet (la gestion concertée d'un espace) et l'outil (la carte) le sont. Outre le scientifique qui accumule les connaissances sur l'espace et l'expert qui conseille les politiques territoriales, nous pensons qu'en effet un troisième métier peut s'affirmer pour le géographe, celui d'un maïeuticien ${ }^{11}$, à la

11. Cf. les propositions de R. Brunet dans le débat qui suit. 
12. Il y faut pour cela des terrains d'expérimentation où I'on a l'occasion d'appuyer le processus de décision territoriale à différentes échelles. croisée des chemins entre le savoir territorial et la pratique territoriale. Il s'agit alors de s'appuyer sur les avantages du concept territorial, espace irremplaçable de débat sur scène et de concertation entre société civile et institutions, pour renforcer le lien entre local et global, qui recoupe aussi pour nous, de façon sous-jacente, la question des rapports entre savoir technique et société civile.

Trois premiers axes d'investigation se dessinent déjà, comme le soulignent nos premières expériences de terrain. D'abord, le renforcement du pouvoir social et politique dans le processus de décision sur les territoires passe par la conception de supports différents d'aide à la décision sur le territoire, permettant un meilleur équilibre entre les connaissances et les influences des services techniques et des acteurs locaux, à quelque échelle que ce soit. Ensuite, une interaction profonde entre les différents niveaux territoriaux doit être mieux appuyée, donc pensée, théorisée et expérimentée. Cela implique autant un investissement théorique qu'une conception méthodologique pragmatique. Pour les avancées théoriques, il s'agira d'investir dans la multiterritorialité, les conditions de son expression, de sa reconnaissance dans les perceptions sur le territoire et de son mode de fonctionnement. Les travaux méthodologiques pourraient s'orienter vers la mise au point d'un processus différent de planification, qui serait " ascendante ", allant du local au global (D’Aquino, 2001) : un processus formalisable d'appropriation territoriale collective, qui débute par une responsabilité effective (territoire politique), puis une régulation concertée (territoire institutionnel), enfin un investissement commun dans l'action et la planification (territoire économique). Ascendant, c'est un processus d'appropriation territoriale qui atteint alors graduellement toutes les échelles territoriales $^{12}$, sans envisager celles-ci comme de simples poupées gigognes emboîtées.

Pour ces trois différents points, de nouveaux supports méthodologiques d'accompagnement aux processus apparaissent nécessaires, dans lesquels le support cartographique, croisé avec d'autres outils, apparaît essentiel. L'enjeu est dans l'interactivité de ces supports, vue comme une interactivité entre le savoir technique et social et entre les différents niveaux territoriaux de concertation et de décision, autant que comme une interactivité entre l'homme et l'outil... La maïeutique géographique: un nouveau savoir de l'interactivité entre les hommes et les territoires?

\section{L'accompagnement géographique d'une territorialisation ascendante}

Toute innovation, pour être intégrée ou pour émerger de la société, nécessite la création d'un environnement favorable (Boserup, 1970; Le Bras, 1995). C’est ce que P. Pélissier (1995, p. 4) évoque dans la "supériorité des structures » et P. Gourou (1973, p. 26) dans les "techniques d'encadrement ". Un premier objectif est de créer localement un terrain institutionnel et comportemental où les acteurs puissent exprimer leurs perceptions, enrichir leurs savoirs, affirmer leurs compétences et peser sur le processus de développement; il porte sur l'environnement politique (la reconnaissance d'un pouvoir local), l'instrumentation technique et administrative (la mise en retrait de ce pouvoir dans le processus local de décision) et la (re)mobilisation sociale des acteurs locaux.

\section{Un nouveau mode d'appropriation territoriale}

Cette approche du territoire relève d'une problématique spécifique, consistant à définir le cadre conceptuel de l'accompagnement d'un processus démocratique ascendant, 
interconnectant les différents niveaux territoriaux pour la gestion de l'espace. C'est la proximité spatiale qui est au cœur de cette spécificité territoriale. Elle n'est nullement synonyme de proximité sociale et identitaire, mais une première étape de la construction d'un "nouveau " territoire, à travers un processus graduel d'émergence d'une identité territoriale, l'appropriation territoriale, qu'il s'agit d'accompagner et de favoriser.

Cette appropriation, pur phénomène de représentation par lequel les groupes humains pensent leur rapport à un espace matériel, ne peut se construire que par l'intégration progressive d'un sentiment local, au travers d'un processus mental collectif (Poche, 1983). On sait que la prise en considération du seul espace vécu a montré ses limites (Di Méo, 1985; Brunet, 1990) et que l'émergence d'une identité territoriale ne peut pas davantage se résumer à une activité économique, sociale ou culturelle commune dépourvue d'existence politique ou administrative reconnue. La seule facette économique n'est ni génitrice, ni constitutive du territoire (Preteceille, 1991). Ce type d'action de l'homme sur l'espace, et les logiques de structuration et d'organisation spatiales qui en découlent, sont plutôt considérés ici comme les produits, et non les origines, de l'«idée » territoriale. Enfin, l'identité territoriale ne se réduit pas davantage à une identité politico-juridique ou à l'enracinement dans un lieu; il y faut un peu de tout cela, et quelque chose de plus: une conscience (Brunet et al., 1992). L'histoire de chaque identification territoriale peut par conséquent être très différente et provenir de phénomènes et d'environnements très variables. C'est pourquoi il est essentiel de réfléchir à un processus croissant d'émergence d'une nouvelle identité territoriale active: l'accompagnement étape par étape d'une dynamique d'appropriation territoriale, par une responsabilisation initiale à l'égard de l'espace, qui aboutira à son appropriation.

Il s'agit ainsi de faire émerger un véritable espace politique local (Lévy, 1991), dont les acteurs se sentent responsables et où se jouent des relations de pouvoir (Bourdieu, 1980) en vue de son appropriation (Raffestin, 1980). "Pour que les suffrages expriment autre chose que les individus, pour qu'ils soient animés dès le principe d'un esprit collectif, il faut que le collège électoral élémentaire ne soit pas formé d'individus rapprochés seulement pour cette circonstance exceptionnelle, qui ne se connaissent pas, qui n'ont pas contribué à se former mutuellement leurs opinions [...]. Il faut au contraire que ce soit un groupe constitué, cohérent permanent [...]. Alors chaque opinion individuelle, parce qu'elle s'est formée au sein d'une collectivité, a quelque chose de collectif » (Durkheim, 1969, p. 138). Premier élément d'une identité plus complète, l'"identité politique " (Lévy, 1994, p. 97) est perçue comme le préalable indispensable à toute velléité de gestion locale décentralisée. L'émergence d'un véritable lieu politique (Lévy, 1994), combinaison de pratiques politiques et d'un contexte local, est donc la première étape, cruciale, du processus d'appropriation territoriale, induite par un sentiment collectif d'un pouvoir possible sur "son " territoire.

Lorsque cette première étape, politique, de l'appropriation territoriale aboutit à des décisions et à des actions communes sur l'espace, on a affaire à un espace gouverné, un espace institutionnalisé, c'est-à-dire un mode d'autorité sur les personnes, les processus ou les relations sociales légitimé collectivement (Sachs, 1986). L'institution n'est pas prise ici selon la définition du courant marxiste mais dans le sens anthropologique d'Eisenstadt (1971), comme tout un ensemble d'actions ou de pratiques organisées de façon stable et reconnues par tous, comme un champ de relations sociales convergentes et légitimées. Elle est définie par des règles, des codes de conduite, des 
13. "L'expression "nos droits sur les ressources" est souvent utilisée pour fonder une identité culturelle " (Sandberg, 1993, p. 323). normes de comportement, et par la manière dont ces conventions sont appliquées (Putnam, 1993; Ostrom et al., 1994; Weber et Reveret, 1993).

Ainsi, après la dimension politique, c'est la dimension institutionnelle d'un futur territoire qui s'affirme, par la reconnaissance collective d'une règle commune sur l'espace, qui constitue la première liaison symbolique forte entre la dimension sociale et la dimension spatiale du futur territoire: notre territoire est d'abord collectivement façonné par des règles communes. L'émergence d'un territoire local est ainsi liée à l'apparition d'une institution collective distincte de l'État-nation et régulant symboliquement une portion locale de l'espace. L'accord sur la règle " fait " ce territoire, qui émerge comme espace d'une institution commune. L'affirmation de cette dimension institutionnelle par l'animation d'accompagnement, en développant les interactions entre individus et entre l'individu et l'espace, en favorisant la reconnaissance du collectif dans le regard de l'autre (pouvoir central, services publics, collectivités locales voisines), contribue ensuite à faire progresser cette identification territoriale qui, comme toute construction identitaire, s'établit par rapport à l'autre ${ }^{13}$. Nous sommes ici proches de la notion de "groupe de localité " de B. Poche (1985), une structure sociale locale composée d'individus qui ont un intérêt si commun à se situer dans un territoire qu'ils en évacuent les conflits qui peuvent les opposer par ailleurs, et qu'ils mettent en acte un certain nombre de comportements pour lesquels la notion de territoire intervient de façon significative.

Ce territoire est donc d'abord politique, puis institutionnel, enfin seulement et partiellement identitaire. Nous appréhendons ici l'appropriation territoriale en tant que construction progressive d'une identification collective active. Selon les cas, elle consiste à transformer un sentiment diffus d'identification territoriale en une responsabilité active, ou à induire une identification à partir d'une prise initiale de responsabilités. Pour cela, elle transforme la proximité spatiale entre individus d'abord en proximité politique (responsabilité d'agir sur le lieu), puis institutionnelle (règle collective sur un lieu), enfin identitaire jusqu'à un certain point (appropriation collective d'un espace physique), pour aboutir à une cohésion sociale essentielle à la dynamique future du nouveau territoire.

L'identité collective est alors indissociable de la dimension politique, c'est-à-dire de l'émergence d'une volonté d'agir au sein d'un collectif (Pizzorno, 1986; Bayart, 1996). La production de territoire est envisagée ici dans le cadre d'une compréhension interactionniste s'appuyant sur les acquis de la psychologie sociale et où la dimension politique est réhabilitée (Raffestin, 1980; Lévy, 1996), l'expression du politique comprenant la dimension institutionnelle et s'intéressant aux processus de production de légitimités sociétales (Lévy, 1991). Adhésion des individus à la fois à un groupe social et à un espace, la prise en charge collective d'un espace local et de son avenir est à l'origine d'un véritable processus de socialisation (Brunet et al., 1992, p. 481), qui associe progressivement à une base matérielle un système de valeurs à significations multiples et combinées (Debarbieux, 1996).

Le fait social total que l'on devine autour du phénomène territorial ne s'affirme de la sorte qu'à ce stade, avec l'expression croissante d'une territorialité, définie comme territoire intégralement socialisé (Gottmann, 1973; Sack, 1986). Avant cela, le fait territorial existe (lieu politique), est opérationnel (champ institutionnel), mais ne constitue pas encore un fait total. Le territoire émerge donc ici d'une construction socio-institutionnelle, arène démocratique qui permet la régulation en une cohérence 
commune de dynamiques locales, toujours diverses, parfois contradictoires (Lévy, 1993). Il n'est donc plus un impératif tenu pour "biologiquement nécessaire ", un ensemble naturel ou spontané dont les frontières s'imposeraient d'elles-mêmes, mais il émerge graduellement. Espace finalisé, il est le résultat jamais identique d'un processus social, dans lequel la légitimation d'une dynamique institutionnelle commune a eu un rôle primordial (Alliès, 1980). La mémoire et l'épaisseur historique (Marié, 1982; Di Méo, 1991) n’y semblent plus essentielles.

Ainsi, l'intérêt croissant pour le "devenir local " (Amphoux et Ducret, 1985) est le signe d'une vitalité et d'une adaptation de la société locale, qui peuvent faire naître une nouvelle identité territoriale. C'est ce qui semble se passer pour les régions administratives françaises, l'activisme de leurs responsables sur ce thème ayant réussi à ancrer en moins de vingt ans le territoire régional dans l'identité collective locale (Dumont, 1997; Dedie et Genieys, 1998) ; et peut-être dans l'apparition d'une identité régionale dans des régions pluriethniques africaines (Pillet-Schwarz, 1997). C'est donc un territoire vivant, changeant, qui peut apparaître ou disparaître au gré des dynamiques sociales. Si la géographie se doit bien de transformer un objet naturel en objet socialisé en produisant de la valeur sociale ajoutée (C. et G. Bertrand, 1992, p. 110), nous sommes bien ici au cœur d'un enjeu géographique. Espace politique, puis espace institutionnel, enfin espace identitaire: fondé sur une "invention précieuse de la territorialité "(Ferrier, 1998, p. 26), ce nouveau territoire est en conséquence à accompagner, dans le cadre d'une "géographie de l'action " (Claval, 1997) qui cherche alors à concevoir de nouvelles méthodes d'intervention.

\section{Vers une nouvelle démarche d'appui territorial, la planification territoriale ascendante}

Qui dit planification, dit projection dans le futur des possibles; et « le rapport aux possibles est un rapport aux pouvoirs" (Bourdieu, 1980, p. 108). La finalité de la planification que nous proposons serait de changer les rapports des différents acteurs locaux aux pouvoirs (et à l'information). Les autres objectifs d'une gestion locale territoriale habituellement mis en avant (développement socio-économique, réglementations collectives, planification locale, préservation de l'environnement, etc.) ne viennent qu'ensuite, lorsque le processus local de gestion a acquis une efficacité sociopolitique et institutionnelle. Les méthodes de planification sont donc ici beaucoup plus des appuis aux processus de décision que des supports de solutions techniques. Dans la lignée d'un géographe précurseur oublié (Webber, 1980), nous nous intéressons particulièrement aux mécanismes d'apprentissage collectif, aux problématiques de la médiation et à une démocratisation plus poussée des processus de décision sur le territoire.

Ce que nous appelons la planification territoriale ascendante vise spécifiquement l'amorce rapide (deux à trois ans) d'une dynamique réellement autonome pour la réflexion collective sur la gestion du territoire, par l'émergence endogène de nouveaux espaces politiques, progressivement à toutes les échelles de prises de décision. Sa finalité n'est donc pas dans la conception d'un "Plan " (planification participative), la mise en œuvre d'innovations et d'organisations économiques (développement local) ou techniques (gestion des ressources naturelles), ni dans la réalisation d'infrastructures socioéconomiques (certaines approches de l'aménagement du territoire et $\mathrm{du}$ développement local). L'hypothèse initiale est que l'installation d'une dynamique locale responsable et autonome pour la gestion du territoire est obligatoire en préalable à toute action de gestion concertée d'un territoire. En particulier, l'exercice de planification ne peut avoir 
14. Les processus endogènes de changement désignent tous les changements sociaux répondant à des dynamiques internes aux sociétés. lieu avant que les populations aient accompli d'elles-mêmes tout le processus endogène de prise de conscience et de concertation sur la nécessité d'abord d'agir ensemble, ensuite de penser au long terme. La préservation et la gestion des ressources ne réussiront localement que lorsque les populations seront sûres d'avoir un réel pouvoir sur leur régulation. Enfin, les décisions d'investissement de la société civile sont difficilement judicieuses avant d'avoir une vision globale du territoire et de son avenir, et ne seront de toutes façons que rarement durables ou efficaces, s'il n'existe pas une instance collective locale fiable de planification et de gestion du territoire.

Comme l'avaient pressenti certains sociologues et géographes urbains américains (Sennett, 1970; P.S. Smith, 1978; Webber, 1980), une planification de bas en haut est davantage un processus, prenant sens dans une séquence temporelle de négociations et d'interactions avec les niveaux englobants, qu'une procédure technique, même si celle-ci s'appelle "plan local de développement ". L'innovation technique, si elle est utile, et plus globalement l'appui-conseil technique, n'est pas considérée ici comme partie intégrante du processus d'appui à la planification ascendante. C'est un appui différent, de toutes façons postérieur à la mise en place du processus de planification, c'est-à-dire à la réalisation d'une dynamique endogène de réflexion et d'action collective locales autonomes du pouvoir technique. La planification territoriale ascendante repose ainsi sur trois hypothèses: le principe d'endogénéité des dynamiques locales, le principe de permanence de la démarche de planification, le caractère normalement ascendant d'une planification continue.

\section{Le concept d'endogénéité}

Le bouleversement profond du comportement et du rôle de l'encadrement technique devient la priorité primordiale, avant même la formation des acteurs locaux. Les acteurs locaux ont déjà beaucoup de compétences instinctives pour la gestion territoriale décentralisée, mais qui sont totalement étouffées par le comportement de l'encadrement technique et administratif. Rien ne sert de multiplier leurs compétences, par la formation ou la décentralisation des pouvoirs, tant que l'environnement local ne leur permettra pas de les exprimer. La phase initiale, où le conseil technique est en retrait, aura permis aux populations d'installer en amont un cadre stratégique de développement local plus axé sur leurs propres aspirations. Le conseil technique pourra ainsi ensuite intervenir, en particulier sur les décisions d'aménagement du territoire et les innovations techniques, en outrepassant moins ses missions. L'intervention de l'expertise technique porte à ce stade sur le " comment ", non sur le "vers quoi ».

Le premier principe de l'endogénéitél1 est donc que l'accompagnement technique ne fixe aucun objectif préalable à sa démarche d'appui, si ce n'est d'être disponible pour une dynamique endogène de prise de décision concertée sur le territoire. Aucune entrée particulière (gestion des ressources naturelles, protection de l'environnement, développement socio-économique, règles collectives) n'est imposée: la démarche se distingue de la plupart des approches participatives, qui interviennent en ayant déjà contraint le sujet d'intervention. De même, aucune exigence méthodologique initiale (diagnostic, planification) n'est prescrite: c'est en s'enferrant dans les voies technicistes du diagnostic "global » et de la planification «intégrée " que le concept participatif a échoué dans sa remise en question de l'interventionnisme de l'encadrement technique sur la société locale. Au contraire, le principe d'endogénéité refuse de considérer ces deux outils comme des étapes initiales obligatoires et imposées. 
Au lieu de quoi, l'objectif primordial est d'appuyer le développement d'une dynamique endogène de prise en charge du territoire, sur quelque sujet et sous quelque forme que ce soit. Les acteurs fixeront ce qu'ils considèrent comme une priorité dans cette nouvelle (re)prise de pouvoir sur leur espace et leurs ressources. Une dynamique voulue et créée localement est par définition incompatible avec un besoin, une analyse, une action auxquels elle est simplement associée. L'approche par l'endogénéité est beaucoup plus optimiste que les démarches participatives sur les capacités d'une société locale à identifier seule les principaux enjeux de développement de son territoire. Si les enjeux considérés comme essentiels par le conseil technique sont réels, ils apparaîtront tôt ou tard aux responsables locaux, mais avec une assise endogène qui leur donnera beaucoup plus de force et de pérennité. L'hypothèse est que, dans la plupart des situations, les priorités locales recouperont graduellement tous les enjeux fondamentaux d'un développement durable, une fois que se sera affirmé un authentique processus endogène et démocratique de prise de décision sur le territoire.

Le deuxième principe de l'endogénéité est que la planification territoriale est avant tout une œuvre politique et non technique. En effet, il n'existe pas d'aménagement neutre de l'espace (Gumuchian, 1991, p. 7) et il a souvent été oublié que la planification est un cadre de réflexion pour les gouvernants, qui leur permet ensuite de s'acquitter avec un maximum d'efficacité de leurs responsabilités d'ordre économique et social (Meynaud, 1963). La décentralisation de l'œuvre de planification ne fait que modifier la nature de ces gouvernants. Dans une telle situation, l'analyse technique, même interdisciplinaire, est toujours partielle et sectorielle, sousévaluant une partie des aspects politiques, des mentalités et des valeurs locales, des forces extérieures et des événements imprévisibles. L'analyse technique ne peut pas proposer d'" hypothèses ", mais des modèles explicatifs qui dirigent la réflexion dès les premiers pas ${ }^{15}$. Face à une situation complexe comportant une large part d'incertitude, même à court terme, toute option technique sous-entend un choix sociétal, éthique ou politique. Une intervention extérieure ne peut avoir la prétention d'intégrer les soubassements sociaux indispensables pour identifier ${ }^{16}$ des actions. Le seul savoir technique ne peut suffire à choisir entre le " rationnel ", l'" équitable ", le "raisonnable", le "prudent ", etc.

Le principe d'endogénéité suppose que l'expertise n'est justifiable que dans sa fonction originelle: restituer de façon la plus neutre possible la connaissance et l'information techniques et trouver une réponse aux questions uniquement techniques que lui pose véritablement la société locale. Devant l'incertitude de l'avenir, la responsabilité de choix politiques et éthiques doit être laissée aux décideurs politiques légitimes, selon le principe d'une démocratie représentative, et aux populations, selon le principe d'une démocratie participative. Le développement local vise l'installation d'un processus local et collectif de prise de décision, ce qui souligne un affaiblissement prononcé du rôle de l'environnement institutionnel, y compris de l'environnement technique, dans l'acte de décider. Si l'enjeu est bien de transformer l'acteur local en décideur local, on ne peut laisser l'expert guider le processus de décision, explicitement ou implicitement.

\section{L'apprentissage continu d'une prise en charge commune du territoire}

Ainsi, face à la variabilité et à l'incertitude du complexe "territoire ", la planification territoriale ascendante ne propose ni des prévisions ni des outils de gestion. Elle ne
15. Ils ont à la fois la prétention d' « expliquer " la complexité et l'intention illégitime de définir les avenirs " raisonnables " à la place des acteurs politiques.

16. Identification dont l'artifice le plus insidieux et le plus courant consiste à proposer plusieurs solutions possibles, en restreignant le champ des possibles à quelques "scénarios" choisis implicitement par l'expert et obéissant évidemment tous au même choix de valeurs... fait par ce dernier. 
17. À la fois droits de pouvoir (de décision, de contrôle, de sanction) et devoirs de gestion (préservation de l'intérêt commun). cherche pas à construire des modèles évaluant les futurs possibles, à débattre de futurs prédéterminés autour de théories explicatives, contrairement à la planification technico-économique. Elle ne consiste pas non plus à définir une politique d'équipement et d'investissements dans l'espace, contrairement à la version habituelle de l'aménagement du territoire et du développement local. Enfin l'enjeu n'est pas celui, utopique (Rölling, 1994), de rendre compte des conditions de la prise de décision dans un univers complexe. La démarche cherche simplement à favoriser un processus collectif de prise de décision qui s'appuie sur une représentation consensuelle et surtout endogène du territoire et de son futur, qui reste à réajuster, voire bouleverser, à chaque étape. Il s'agit en définitive d'aider les participants à parvenir à une zone d'"ignorance fructueuse" (Favereau, 1994).

Pratiquement, le principe d'endogénéité implique que, dans une "dynamique locale ", la participation des populations ne se limite pas à un apport physique ou financier, ni à une consultation régulière, ni même à une association à une prise de décision, mais à une prise en charge directe, dès les premières étapes, du processus collectif de prise de décision. Dès le départ, le processus doit être le plus indépendant possible d'une animation extérieure, aussi bien à cause des comportements instinctivement interventionnistes de tout encadrement technique que pour un apprentissage de la responsabilité, qui ne peut se faire que par la prise effective de responsabilité. Toutes les étapes du processus de décision doivent par conséquent être sous la responsabilité17 réelle des acteurs locaux, depuis l'adoption des modes de concertation et de prise de décision internes jusqu'au choix des différentes actions et de leurs méthodes de réalisation.

La planification territoriale ascendante est donc indissociable d'une démarche d'apprentissage. La théorie bourdieusienne de la pratique rappelle que les objets de connaissance sont construits, et non passivement intégrés (Bourdieu, 1980, p. 87) et que la lente modification des habitus, le changement permanent de la dynamique des champs de forces nécessitent une lente maturation que seule peut donner une démarche d'apprentissage. Cette forme de planification, conçue comme l'accompagnement d'un apprentissage, ne peut donc être une action réduite dans le temps, à l'inverse de la planification locale participative. C'est un appui permanent à un processus qu'il s'agit d'installer durablement dans l'environnement de la société locale. $\mathrm{Au}$ lieu d'un produit circonstanciel ou d'une impulsion initiale, son objectif est d'assurer une offre locale d'appui qui amorce, accompagne et soutienne les processus d'apprentissage nécessaires à l'environnement institutionnel et aux acteurs locaux. À partir d'une entrée privilégiée, la collectivité locale de base et son champ territorial, la démarche cible expressément l'intégration progressive, ascendante, de la totalité des différents acteurs et institutions concernés. C'est ainsi une démarche d'autorégulation ascendante, l'" auto " faisant ici référence à l'autonomie sociétale vis-à-vis du technique et non à l'autonomie d'un niveau de décision donné, puisque, à l'inverse, c'est leur interdépendance qu'elle postule. Il serait de ce fait peut-être préférable de nommer cela endorégulation plutôt qu'autorégulation.

Comment dans ces conditions parvenir à organiser un appui cohérent, face à des enjeux géographiques et sociopolitiques s'exprimant différemment aux différents niveaux de l'architecture multiterritoriale? La voie choisie passe par la mise en route, dans les territoires locaux, d'un processus démocratique de concertation et de prise de décision qui se charge progressivement d'un exercice permanent de planification, continu dans le temps et remontant graduellement l'architecture territoriale des 
niveaux de décision. Elle cherche à aider à inscrire ce processus démocratique local dans une diversité d'espaces, du local au mondial, et une diversité de temps, du court terme au très long terme (Calame, 1994, p. 56). Le concept d'ascendance (D'Aquino, 2001) suggère donc une dynamique tridimensionnelle (société, espace, temps), diffusant à partir de la collectivité de base vers le reste de l'environnement sociétal, vers les niveaux géographiques englobants et vers le futur pour la continuité du processus de décision. La stratégie de la démarche est d'appuyer le développement d'une conscience et d'une compétence locales pour une dynamique démocratique de planification territoriale, et de l'aider ensuite à s'affirmer aux niveaux englobants. Ce n'est donc pas le projet global à un temps $t$ d'une collectivité sur un territoire qui est recherché, mais la mise en route d'une dynamique graduelle, donc incomplète. Elle passe par l'apprentissage, entre les différents niveaux, d'une gestion concertée du territoire qui respectera les enjeux et les points de vue de chaque niveau.

L'objectif de la planification territoriale ascendante ne sera cependant jamais de produire un mode d'organisation de référence pour la prise de décision collective sur un territoire. Elle constitue un apprentissage collectif par l'action, processus génétique libre de constituer de lui-même des formes toujours nouvelles d'organisation, de régulation et de complémentarité entre niveaux de décision, adaptées à chaque situation. En termes méthodologiques, l'objectif de la démarche sera donc plutôt de concevoir une méthode de référence (l'accompagnement) pour aider à amorcer et enrichir des dynamiques sociopolitiques de prise en charge d'un territoire. L'objectif est d'accompagner graduellement un processus démocratique de décision multiscalaire et ascendant, de quelque forme qu'il soit.

La plate-forme méthodologique n'aura alors pour ambition que de rassembler des supports didactiques appuyant l'émergence de situations territoriales propices à l'expression du changement social, de collectivités locales capables ${ }^{18}$ d'assumer leur propre processus continu de planification, et d'un encadrement technique qui ne cherche pas à être le guide du processus de décision sur le territoire. Le rôle de la connaissance scientifique est d'améliorer à chaque instant les conditions de prises de décision des responsables légitimes, non de proposer des orientations, dont l'enjeu dépasse toujours la sphère technique.

Il ne s'agit surtout pas d'identifier des itinéraires, autrement dit des "scénarios " permettant d'atteindre ces futurs utopiques. Comme son emploi cinématographique le souligne, le scénario est un chemin connu vers une fin programmée, dont toutes les étapes sont maîtrisées, justement grâce à sa construction. Ce n'est pas le parti pris de la démarche, qui considère à l'inverse que la complexité de la situation ne permet pas à l'expertise de deviner les chemins possibles pour atteindre des futurs de toutes façons imaginaires. Ainsi, contrairement à la plupart des démarches de planification, même dites participatives, la planification territoriale ascendante considère les différents appuis (diagnostic participatif, plan local de développement, plan d'aménagement de l'espace, schéma directeur, plan d'occupation des sols, etc.) comme tous imparfaits et transitoires, à reprendre et à enrichir dans les phases ultérieures d'un processus itératif autonome, au gré des attentes locales s'exprimant progressivement.

Cette affirmation de l'incomplétude et de la relativité de tous les outils possibles de planification est certes en contradiction avec la nature holistique et systémique du développement territorial, mais cela permet de construire une approche progressive, plus opérationnelle, pour l'apprentissage et la gestion de la complexité. Appréhender toute la
18. « C'est-à-dire des représentants (i) qui sont pleinement conscients du poids des responsabilités qui leur sont confiées; (ii) dont les attributions sont reconnues et appuyées (pas seulement dans les textes) par tous les services, par l'administration et les populations; (iii) qui ont accès à I'information et à la connaissance indispensables pour prendre leurs décisions; (iv) qui ont acquis les compétences nécessaires pour mener un processus collectif de prise de décision " (D'Aquino, 1998, p. 7). 
complexité relève du fantasme. La pratique planificatrice ne peut être qu'un processus, non un produit fini. Comme toute pratique, elle se construit dans le temps et reçoit de celui-ci à la fois sa forme et son sens (Bourdieu, 1980, p. 167). Le travail de réflexion collective sur l'avenir d'un territoire est un travail d'action plutôt que de prédiction.

\section{Conclusion}

En contradiction apparente avec les préceptes d'un développement intégré, la planification territoriale ascendante représente donc l'option pour une planification complétive plutôt que globale, qui se fonde sur notre incapacité à formaliser a priori une programmation judicieuse pour améliorer une situation complexe. Cette nouvelle approche de l'appui à la planification réalise graduellement un enrichissement de la réflexion et de l'action collective au rythme endogène de la société locale. Nous revenons en cela aux mobiles originels de la démarche du développement local et à ses meilleures applications pratiques, de Paulo Freire ${ }^{19}$ aux méthodes du GERDAL (Darré, 1993; Ruault, 1996) et à certaines expériences de planification décentralisée (Godard et Céron, 1985). Elle est proche aussi d'une interprétation contemporaine et institutionnaliste du concept français d'aménagement du territoire, qui ne se préoccupe plus de prévoir des actions dans le temps, mais de créer, par des politiques institutionnelles, des "contextes d'action " (Padioleau in Némery et Wachter, 1994, p. 29). Enfin, nous sommes dans la même perspective que la nouvelle prospective en continu (Bailly, 1999).

La construction méthodique de supports pour l'action reste d'actualité pour une géographie qui accepte cette intentionnalité politique et qui ne recourt pas abusivement à l'autorité de la science pour identifier le "bien commun » à la place du nécessaire débat démocratique (Mercier, 1989, p. 71). Dans ce cas, même si nous avons tenté d'en poser certains principes, la construction d'un appui global et opérationnel, d'outils et de supports spécifiques, est toujours à poursuivre. Il passe par l'expérimentation dans l'action.

19. Au début des années 1960 en Amérique latine, la Participatory Action Research (PAR) de Paulo Freire est une démarche d'essence politique qui veut organiser une planification autonome des populations. Les agents extérieurs y jouent un simple rôle d'animateur de réunions, de catalyseur et de facilitateur, et accompagnent les acteurs locaux pendant une longue période de renforcement de leur confiance en soi et de leur poids politique, sans devoir produire des données et des résultats pour un projet défini et limité dans le temps (Chambers, 1993).

\section{Références}

AllaiRE G. (1988). «Problèmes méthodologiques de l'analyse localisée des systèmes socioéconomiques », p. 179-190, in M. JOLlivet dir., Pour une agriculture diversifiée, Paris: L'Harmattan, coll. «Alternatives rurales », $335 \mathrm{p}$.

ALLİ̀s P. (1980). L'Invention du territoire. Grenoble: Presses universitaires de Grenoble, 184 p.

Amphoux P., Ducret A. (1985). "La mémoire des lieux ». Cahiers internationaux de sociologie, t. LXXIX, p. 197-202.

BADIE B. (1994). Le Développement politique. Paris: Economica, XI, $223 p$.

BAdIE B. (1997). «Entretien ». Sciences humaines, Hors série n 17, p. 63-68.

BAilly J.-P. (1999). Demain est déjà là. Prospective, débat, décision publique. La Tour d’Aigues: Éd. de l'Aube, $166 \mathrm{p}$.

Balme R. dir. (1996). Les Politiques du néo-régionalisme. Paris: Economica, 301 p.

Balme R., Faure A., Mabileau A. dir. (1999). Les Nouvelles Politiques locales. Paris: Presses de Sciences Po, coll. «Dynamiques de l'action publique», $486 \mathrm{p}$.

BARBER B. (1997). Démocratie forte. Paris: Desclée de Brouwer, 320 p.

BAYART J.-F. (1996). L'Illusion identitaire. Paris : Fayard, 306 p.

Benko G., LiPIETZ A. (1992). Les Régions qui gagnent. Paris: Presses universitaires de France, 424 p. 
Bertomé J., Mercolret J. (1993). Méthode de planification locale pour les organisations paysannes d'Afrique sahélienne. Paris: L'Harmattan, $349 \mathrm{p}$.

BIERSCHENK T. (1988). «Development project as arenas of negociation for strategic groups. A case study from Benin ». Sociologia ruralis, t. XXVIII, nº 2-3, p. 146-160.

Boltanski L., Thévenot L. (1991). De la justification: les économies de la grandeur. Paris: Gallimard, $485 \mathrm{p}$.

Bonnemaison J., CAMBrézy L., QuintY-BouRgeols L., dir. (1997). Le Territoire, lien ou frontière. Paris: ORSTOM, 2 vol., $315+266$ p.

Boserup E. (1970). Évolution agraire et pression démographique. Paris: Flammarion, coll. "Nouvelle bibliothèque scientifique », $218 \mathrm{p}$.

BouRdieu P. (1980). Le Sens pratique. Paris : Éd. de Minuit, 477 p.

BouRdieu P. (1994). Raisons pratiques. Sur une théorie de l'action. Paris: Le Seuil, 251 p.

BRUNET R. (1990). Le Territoire dans les turbulences. Montpellier: RECLUS, coll. « Géographiques », $223 \mathrm{p}$.

Brunet R., FerRas R., Théry H. (1992). Les Mots de la géographie. Dictionnaire critique. Paris/ Montpellier: La Documentation française/RECLUS, coll. « Dynamiques du territoire », 518 p.

Calame P. (1994). Un Territoire pour l'homme. La Tour d'Aigues: Éd. de l'Aube, 93 p.

ChAMBERS R. (1993). "Methods for analysis by farmers: the professional challenge ». Journal for Farming Systems Research-Extension, vol. 4, nº 1, p. 87-101.

Chambers R., PACEY A., Thrupp L.A. (1989). Farmer first: farmer innovation and agricultural research. Londres: Intermediate Technology Publications. Les Paysans d'abord: les innovations des agriculteurs et la recherche. Paris : Karthala, $346 \mathrm{p}$.

Chauveau J.-P. (1994). « Participation paysanne et populisme bureaucratique. Essai d'histoire et de sociologie du développement », in J.-P. JACOB, P. LAvigne-Delville, Les Associations paysannes en Afrique, APAD, Association euro-africaine pour l'anthropologie du changement social et du développement. Paris: Karthala, IUED, Institut universitaire d'études du développement, 307 p.

Claval P. (1997). "Le territoire dans la transition à la postmodernité ", in J. Bonnemaison, L. Cambrézy, L. QuinTY-Bourgeols, dir.

Clouet Y. (1993). Bilan des expériences de gestion de terroir et des ressources naturelles. Montpellier: CIRAD, Observatoire du Sahara et du Sahel, 79 p.

Conen J. (1989). "Deliberative and democratic legitimacy », in A. Hamlin, Ph. Petit dir., The Good Polity, normative analysis of the state. Oxford: Blackwell.

Commère R., dir. (1989). Le Développement local en milieu rural. Saint-Étienne: Université, 221 p.

Courlet C. (1994). « Les systèmes productifs localisés, de quoi parle-t-on? » in C. CourLet, B. SoulaG dirs., Industrie, territoires et politiques publiques. Paris: L'Harmattan, $315 \mathrm{p}$.

DAHL R. (1971). Qui gouverne? Paris: Armand Colin, 369 p.

D’Aquino P. (1998). Décentralisation et gestion locale des ressources. L'opération pilote sur les plans d'occupation et d'affectation des sols pour la vallée du fleuve Sénégal. Rapport de présentation de la démarche. Montpellier: Centre international de recherche agronomique pour le développement (CIRAD), $31 \mathrm{p}$.

D'Aquino P. (2000). La Gestion décentralisée des ressources renouvelables en Afrique de l'Ouest: pour une animation inter-institutionnelle plutôt qu'un appui direct à la planification locale. Paris: Association européenne des Instituts de recherche et de formation en matière de développement (EADI).

D’Aquino P. (2001). «Ni planification locale, ni aménagement du territoire: pour une nouvelle approche de la planification territoriale». À paraître, Géographie, économie, sociétés, t. $3, \mathrm{n}^{\circ} 2$.

D’Aquino P., SECK S.M., CISSOKHo A. (1999). « De l'irrigation administrée à une gestion concertée du territoire irrigable: le POAS, une démarche pour une évolution des modes de prise de décision », in Synthèse des résultats du projet PSI-CORAF. Dakar : CORAF, 14 p.

DARRÉ J.-P. (1993). Du sens aux actes. Comment transformer en idées neuves les dialogues de tous les jours ? Paris: Gerdal, Groupe d'expérimentation et de recherche pour un développement agricole local, $234 \mathrm{p}$. 
DeBARBieuX B. (1996). « Le lieu, fragment et symbole du territoires ». Espaces et sociétés, nº 82-83, p. 13-35. DediE O., GENIEYS W. (1998). «Le développement local face à l'Europe : l'invention du pays cathare ». Sciences de la Société, $n^{\circ}$ 45, p. 103-117.

Dı MÉo G. (1985). "Les formations socio-spatiales ou la dimension infra-régionale en géographie ». Annales de géographie, $n^{\circ}$ 526, p. 661-689.

Dı MÉo G. (1991). "La genèse du territoire local: complexité dialectique et espace-temps ». Annales de Géographie, $n^{\circ}$ 559, p. 273-294.

Dı MÉo G. (1998). Géographie sociale et territoires. Paris : Nathan Université, 320 p.

Dumont G.F. (1997). « Le dessein identitaire des régions françaises », in J. Bonnemaison, L. CambrézY, L. QUINTY-BOURGEOIS, dir.

Durkheim M. (1969). Leçons de sociologie. Paris: Presses universitaires de France, 244 p.

DuVERNAY J., dir. (1989). Le Local en action. Paris: Éditions de l'épargne / Association nationale pour le développement local et les pays (ANDLP), Institut de formation en développement économique communautaire (IFDEC), $280 \mathrm{p}$.

EISENSTADT S. (1971). Social Differenciation and Stratification. Londres: Scott, Foresman.

EISENSTADT S. (1983). Tradition, change and modernity. Malabar (USA) : Robert E. Krieger, 367 p.

ELA J.-M. (1987). L'Afrique des villages. Paris: Karthala, 228 p.

FAVEREAU 0. (1994). «Règle, organisation et apprentissage collectif: un paradigme non standard pour trois théories hétérodoxes ", in OrLÉAN A., dir., Analyse économique des conventions. Paris: Presses universitaires de France, p. 115-137.

FerRIER J.-P. (1998). Le Contrat géographique ou l'habitation durable des territoires: Antée 2. Lausanne: Payot, $247 \mathrm{p}$.

Friedberg E. (1993). Le Pouvoir et la règle. Dynamique de l'action organisée. Paris: Le Seuil, coll. «Sociologie », $405 \mathrm{p}$.

Gallais J. (1976). "De quelques aspects de l'espace vécu dans les civilisations du monde tropical ». L'Espace géographique, t. 5, nº 1, p. 5-10.

Godard O., CÉRon J.-P. (1985). Planification décentralisée et modes de développement. L'expérience du Bureau méridional de planification agricole en Provence. Paris: Maison des sciences de l'homme, $205 \mathrm{p}$.

GontCHAROFF G. (1996). « Décalages. Quoi de neuf après trente ans? » Territoires, janvier, p. 18-21.

Gotтmann J.-M. (1973). The Significance of Territory. Charlottesville: University Press of Virginia, $169 \mathrm{p.}$

Gourou P. (1973). Pour une géographie humaine. Paris: Flammarion, coll. " Nouvelle bibliothèque scientifique », $388 \mathrm{p}$.

Hermet G., Badie B., Birnbaum P., Braud P. (1994). Dictionnaire de la science politique et des institutions publiques. Paris: A. Colin, $287 \mathrm{p}$.

HouéE P. (1996). Les Politiques de développement rural. Paris: INRA/Economica, 136 p.

Human P., ZaAiman A. (1996). Gérer pour l'auto-suffisance. Sur l'efficacité des organisations en Afrique. Dakar: Institut de Gorée, 198 p.

Huntington S. (1968). Political Order in Changing Societies. New Haven: Yale University Press, XI$488 \mathrm{p}$.

Katz A. (1993). Self-help in America: A Social Perspective. New York : Twayne.

LASSALE J.-P. (1991). La Démocratie américaine; anatomie d'un marché politique. Paris: A. Colin, $373 \mathrm{p}$.

Le Bras C. (1995). Économie de l'innovation. Paris: Economica, 112 p.

LÉvY J. (1991). Géographies du politique. Paris: Presses de la FNSP, 220 p.

LÉVY J. (1993). «A-t-on encore (vraiment) besoin du territoire? » EspacesTemps, n 51-52, p. 102-142.

LÉVY J. (1994). L'Espace légitime: sur la dimension géographique de la fonction politique. Paris: Presses de la FNSP, $442 \mathrm{p}$.

LÉvy J. (1996). Le Monde pour cité. Paris: Hachette, coll. «Questions de politique », 143 p.

MAGASSOUBA M. (1984). « La participation en milieu rural dans un pays en voie de développement: les communes rurales du Sénégal ». Annuaire du Tiers-Monde 1982-1983. 
Manin B. (1985). «Volonté générale ou délibération? Esquisse d'une théorie de la délibération ». Le Débat, $\mathrm{n}^{\circ} 33$.

MARCH J.-G., OLSEN J.-P. (1990). Rediscovering institutions, the organizational basis of politics. New York: The Free Press, VII-227 p.

MARIÉ M. (1982). Un Territoire sans nom, pour une approche des sociétés locales. Paris: Librairie des Méridiens, $176 \mathrm{p}$.

MengIN J. (1989). Guide du développement local et du développement social. Paris: L'Harmattan, $164 \mathrm{p}$.

MénY Y. (1992). «La république des fiefs ». Pouvoirs, numéro spécial, « La décentralisation ».

MERCIER G. (1989). «Offre et demande sociale ». EspacesTemps, n ${ }^{40-41, ~ p . ~ 69-71 . ~}$

MeYnaud J. (1963). Planification et politique. Lausanne: Études de science politique, $191 \mathrm{p}$.

Miller D. (1992). «Deliberative democracy and social choice ». Political studies, $\mathrm{n}^{\circ} 40$.

Mongolfier J. de, Natal J.-M. (1987). Le Patrimoine du futur. Paris : Economica, 248 p.

NÉmerY J.-C., Wachter S. (1994). Gouverner les territoires. La Tour d'Aigues: DATAR/Éd. de l'Aube, coll. « Monde en cours », $206 \mathrm{p}$.

NGUINGUIRI J.-C. (1998). «Approche participative et développement local en Afrique subsaharienne: faut-il repenser la forme contemporaine du modèle participatif? ", Arbres, forêts et communautés rurales, $\mathrm{n}^{0}$ 15-16, p. 44-48.

Okali C., Sumberg J., Farrington J. (1994). Farmer Participatory Research, Rhetoric and Reality. Brighton : Intermediate Technology Publications, ODI.

Olivier de Sardan J.-P., PAQuot E. (1991). D'un savoir à l'autre. Les agents de développement comme médiateurs. Paris: Ministère français de la Coopération (GRET)/La Documentation française, $204 \mathrm{p}$.

Ostrom E., Gardner R., Walker J. (1994). Rules, Games and common-pool Resources. Ann Arbor: The University of Michigan Press, $369 \mathrm{p}$.

PeCQueUR B. (1989). Le Développement local: mode ou modèle. Paris: Syros, 139 p.

PélISSIER P. (1995). Campagnes africaines en devenir. Paris: Arguments, 318 p.

Pellegrino P., ludi J.-C., Albert G., Castella C., Levy A., Klaue K., Martignon J.-P. (1983). Identité régionale et représentations collectives de l'espace. Actes du colloque de Berne. Berne: Centre régional universitaire de Berne, $181 \mathrm{p}$.

Pijnenburg B., Cavane E. (1997). Community Participation and the Use of PRA in the LDF-Nampula Project. Maputo: FAEF, UEM, Evaluation report.

Pillet-Schwartz A.-M. (1997). « Le temps des régions pluri-ethniques. Un exemple identifié au NordEst du Burkina Faso », in J. Bonnemaison, L. Cambrézy, L. QuintY-Bourgeols.

Pizzorno A. (1986). "Sur la rationalité du choix démocratique », in P. Birbaum et J. LeCA, Sur l'individualisme. Paris: Presses de la FNSP, p. 119-138.

Pizzonno A. (1990). «Considérations sur les théories des mouvements sociaux ». Politix, $\mathrm{n}^{\circ} 9$.

Poche B. (1983). « La région comme espace de référence identitaire ». Espaces et sociétés, nº 42, p. 2-10.

Poche B. (1985). "Une définition sociologique de la région? » Cahiers internationaux de la sociologie, t. LXXIX, p. 226-238.

Preteceille E. (1991). " Paradoxes politiques des restructurations urbaines. Mondialisation de l'économie et localisation du politique ». Espaces et sociétés, nº 59, p. 5-36.

Pretty J., Guit J., Thompson J. Scoones I. (1995). Participatory Learning in Action. A Trainers' Guide for Participatory Learning in Action. Londres: International Institute for Environment and Development (IIED).

Putnam R.D. (1993). Making Democracy Work: Civic Traditions in Modern Italy. New York: Princeton University Press, XV-258 p.

RAFFESTIN C. (1980). Pour une géographie du pouvoir. Paris: LITEC, coll. « Géographie économique et sociale », $249 \mathrm{p}$.

ReINTJES C., HAVerkort B., Waters-BAyer A. (1995). Une agriculture pour demain: une introduction à l'agriculture durable avec peu d'intrants externes. Paris: Karthala, $473 \mathrm{p}$. 
Remy J. (1988). "Les courants fondateurs de la sociologie urbaine américaine: des origines à 1970 ». Espaces et sociétés, n 56, p. 7-38.

Rölling N. (1994). "Platforms for decision-making about ecosystems ", in L.O. Fresco, L. Stroosnijder, J. Bouma, H. Van Keulen, dir., The Future of the Land: Mobilising and Integrating Knowledge for Land Use Options. Londres : John Wiley, p. 385-393.

Rondin J. (1985). Le Sacre des notables: la France en décentralisation. Paris: Fayard, $331 \mathrm{p.}$

Rosenau J.N. (1990). Turbulence in World Politics. A Theory of Change and Continuity. Princeton: Princeton University Press, $480 \mathrm{p}$.

Rosenau J.N. (1992). Governance without government: Order and Change in World Politics. New York: Cambridge University Press, $311 \mathrm{p}$.

Rousseau D. (1996). "De la démocratie participative à la démocratie continue ». Le Monde, $1^{\text {er février, p. } 16 .}$

Ruault C. (1996). L'Invention collective de l'action. Initiatives de groupes d'agriculteurs et développement local. Paris: L’Harmattan, $256 \mathrm{p}$.

SACK R.D. (1986). Human Territoriality: its Theory and History. New York: Cambridge University Press, $256 \mathrm{p}$.

SANDBERg A. (1993). "Gestion des ressources naturelles et droits de propriété dans le grand nord norvégien: éléments pour une analyse comparative ». Nature, sciences, sociétés, t. $2, \mathrm{n}^{\circ} 4$, p. 323-335.

SAndel M.J. (1984). "The procedural democracy and the unemcombered self». Political Theory, $n^{\circ} 12$.

Schönhuth M., Kievelitz U. (1994). Les Méthodes de recherche et de planification dans la coopération au développement: diagnostic rapide en milieu rural, diagnostic participatif. Eschborn: GTZ.

Sennet R. (1970). The Uses of Disorder. Personal identity and City Life. New York: A. Knopf, 198 p.

Tonneau J.-P., Caron P., Clouet Y. (1998). «L'agriculture familiale au Nordeste (Brésil). Une recherche par analyse spatiale ». Nature, sciences, sociétés, vol. 5, n 3 , p. 39-49.

Uphoff N.T., Chen J.M., Goldsmith A.A. (1979). Feasibility and Application of Rural Development Participation: A State-of-the-art Paper. Ithaca: Cornell University, Center for International Studies.

VABI M.B. (1998). «Problèmes liés à l'utilisation des méthodes participatives: enseignements tirés de l'application sur le terrain des PRA/RRA dans certains pays de la sous-région de l'Afrique Centrale ». Bulletin Arbres, Forêts et Communautés rurales, n 15-16, p. 49-55.

VAchon B. (1993). Le Développement local. Théorie et pratique. Québec: Gaëtan Morin, $331 \mathrm{p}$.

Veltz P. (1994). Des territoires pour apprendre et innover. La Tour d'Aigues: Éd. de l'Aube, $94 \mathrm{p.}$

Veltz P. (1997). Mondialisation, villes et territoires. L'économie d'archipel. Paris: Presses universitaires de France, $262 \mathrm{p}$.

Water-Bayer A., Bayer W. (1995). Planification avec des pasteurs. MARP et au-delà, un compte rendu de méthodes centré sur l'Afrique. Göttingen: GTZ, $186 \mathrm{p}$.

WeAver C. (1983). « Le développement à la base: vers une doctrine du développement territorial », in B. Planque, dir. Le Développement décentralisé. Dynamique spatiale de l'économie et planification régionale. Paris: LITEC, p. 179-186.

Webber M.M. (1980). "A different paradigm for planning », in W.R. Burchell, G. SternLieb dir., Planning theory in the 1980's. New Brunswick: Center for urban policy research, p. 151-162.

Weber J., Reveret J.-P. (1993). "Biens communs: les leurres de la privatisation ». Le Monde diplomatique, coll. «savoirs ", $\mathrm{n}^{\circ} 2$.

WeBer J., ReVERET J.-P. (1993). «La gestion des relations sociétés-natures: modes d'appropriation et processus de décision ». Le Monde Diplomatique, coll. « savoirs », $n^{\circ} 2$.

WHYTE W.F. (1981). Participatory approaches to agricultural research and development: a state-ofthe-art paper. Ithaca: Cornell University, Center for International Studies.

Yzerbyt V., Sharon G. (1996). Connaître et juger autrui: une introduction à la cognition sociale. Grenoble : Presses universitaires de Grenoble, 275 p. 